\title{
A Novel Current Calculation Method Based on Temperature Rise of Current-Carrying Conductor
}

\author{
Sen Lv iD and Ming Zong (iD \\ School of Electrical Engineering, Shenyang University of Technology, Shenyang 110870, China \\ Correspondence should be addressed to Ming Zong; ming_zong@163.com
}

Received 26 April 2020; Accepted 14 September 2020; Published 24 September 2020

Academic Editor: Fausto Arpino

Copyright (c) 2020 Sen Lv and Ming Zong. This is an open access article distributed under the Creative Commons Attribution License, which permits unrestricted use, distribution, and reproduction in any medium, provided the original work is properly cited.

\begin{abstract}
Smart grid is the inevitable trend of power grid technology development. As an important terminal apparatus in the power grid, the overload protection method of bimetal used by traditional miniature circuit breakers (MCBs) is difficult to meet the requirements of smart grid. Bimetal uses the thermal effect of current for overload protection, so in this paper, the thermal effect is used to calculate the current. Also, all current carrying conductors except superconductors will generate heat, and the heat will not be saturated, which can meet the characteristics of miniature circuit breakers with small volume and large current. Compared with other current calculation methods, the proposed method has simple structure and wide measurement range, which is an effective way to solve the problem of intelligent MCBs. After derivation, the algorithm in this paper is not affected by the convection coefficient. The experimental results show that the error of the algorithm is less than $5 \%$.
\end{abstract}

\section{Introduction}

An important feature of smart grid is that it can monitor the important parameters (such as voltage and current of each node) in real time, which is helpful for people to make fast and accurate fault diagnosis, response, and accurate load control. As an important terminal apparatus in power grids, miniature circuit breakers (MCBs) must be intelligent to meet the requirements of a smart grid. Current calculation has become an important part of this process.

A current sensor based on magnetic fluid and fiber loop device ringdown technology was proposed in [1], which a sensitivity of $3.3 \mathrm{~mA} / \mu$ s can be realized. A wide linearity range current sensor based on piezoelectric effect was proposed in [2], and it can possess an excellent linear relationship between the output voltage and the AC in the range of $0 \sim 500 \mathrm{~A}$ with the sensitivity of $1.52 \mathrm{mV} / \mathrm{A}$. An AMR-based three-phase current sensor for smart grid applications was proposed in [3]. The obtained experimental results showed that the proposed device could be used to measure current in three-phase electric distribution substations, ensuring a level of uncertainty compatible with smart grid applications (e.g., digital protections). A novel technique for high-frequency contactless current sensing using magnetoresistor sensors with planar magnetic concentrators utilizing conductive materials was proposed in [4]. In addition, there were fluxgate current sensor with a U-shaped magnetic gathering shell; passive electric current sensor based on ferromagnetic invariant elastic alloy, piezoelectric ceramic, and Permalloy yoke; bus bar current sensor with frequency compensation, temperature-compensated magnetostrictive current sensor based on the configuration of dual fiber Bragg gratings; a stray current sensor based on an all-side cylindrical spiral fiber; high temperature and vibration robustness all-fiber current sensor with a fiber-loop architecture and reflection scheme; birefringence variation independent fiber-optic current sensor using real-time SOP measurement; a photonic transducer-based optical current sensor using back-propagation neural network; a novel low-cost multicoil-based smart current sensor for three-phase current sensing of overhead conductors; and so on [5-13].

Although there are many kinds of current measuring devices, they are mostly based on the current magnetic effect. 
The common feature of this kind of current measuring device is that it has a core. When the current is large, the core will be saturated and the measuring range will be reduced. Optical current measurement devices are also widely used, but it is usually large in volume. However, because of its small size and large current, it is difficult to use current magnetic effect or optics to measure current, which restricts the intelligent process of MCB. Therefore, a current measuring device with simple structure and wide measurement range is urgently needed.

Thermal effect is an important property of current. The current flowing through any conductor (except superconductor) will cause the conductor to heat up. There is no saturation problem in the current thermal effect, and the volume of the heating conductor can be optimized according to the volume of MCB. In fact, bimetal uses the thermal effect of current for overload protection in traditional MCBs. Therefore, this paper uses current thermal effect for current calculation, which can be called electrothermal current calculation method based on temperature rise. In order to study the method, a measuring cavity is designed. The algorithm fully considers the influence of convection coefficient, which makes it more universal. The electrothermal current calculation method lays a foundation for the intellectualization of MCBs.

\section{Measuring Cavity and Current Calculation Method}

In order to study the electrothermal current calculation method, a measuring cavity (shown in Figure 1) is designed. Conductor and silicone grease are wrapped by shell and metal plate to form measuring cavity. The environment of heating, heat accumulation, and heat dissipation is simple, which is beneficial to the establishment of equations.

The metal plate and the conductor are made of copper, and the shell material is ABS. As shown in Figure 1(b), the length of the cavity is $40 \mathrm{~mm}$, and the width and height are $20 \mathrm{~mm}$. The thickness of the metal plate and conductor is $1 \mathrm{~mm}$, and the thickness of internal air is $13 \mathrm{~mm}$. When the conductor flows through the current, Joule heat is generated, and heat is emitted through the shell and metal plate. Thermal conductive silicone grease is evenly coated underneath and on both sides of the conductor in order to rapidly transmit heat to the outer shell and then emit it through the air. The thermal circuit model of the cavity is established, as shown in Figure 2.

Because the cavity is symmetrical with respect to $X O Y$, the thermal resistance of the shell and convective thermal resistance in both directions of the $Z$-axis are equal, i.e., the thermal resistances in the $+Z$ and $-Z$ axes in Figure 2 are exactly the same. Also, because the cavity is symmetrical with respect to $Y O Z$, the thermal resistance of the shell and convective resistance in both directions of the $X$-axis are equal; that is, the thermal resistances in the $+X$ and $-X$ axes in Figure 2 are exactly the same. Secondly, the width and height of the cavity are equal, and the heat dissipation area of the $Y$ and $Z$ axes are equal, so the convective thermal resistances of the $Y$ and $Z$ axes are equal. The meanings of the symbols in Figure 2 are shown in Table 1.

The simplified thermal circuit is shown in Figure 3. In the figure, " $a$ " is the conductive thermal resistance along the $X$ axis, " $b$ " and " $c$ " are the conductive thermal resistance along the $Y$-axis, " $d$ " is the conductive thermal resistance along the $Z$-axis, " $e$ " is the convective thermal resistance along the $X$ axis, and " $f$ " is the convective thermal resistance of the other four directions.

It can be seen from Figure 3 that

$$
\begin{aligned}
i_{T}+i_{C} & =q, \\
\frac{u_{C}}{R_{T}}+C \frac{\mathrm{d} u_{C}}{\mathrm{~d} t} & =q .
\end{aligned}
$$

By solving differential equations (2) and (3), the following can be obtained:

$$
u_{C}=q \cdot R_{T}\left(1-e^{-\left(t / R_{T} \cdot C\right)}\right) .
$$

And because

$$
C=\rho c V
$$

the heat generated by the current $I$ flowing through the resistance $R$ is

$$
q=I^{2} R
$$

Therefore, the expression of the temperature rise of the conductor can be obtained:

$$
\theta=I^{2} R \cdot R_{T}\left(1-e^{-\left(t / R_{T} \cdot \rho C V\right)}\right),
$$

where $\theta$ denotes the conductor temperature rise; $I$ denotes the current; $R$ denotes the conductor resistance; $R_{T}$ denotes the total thermal resistance; and $\rho, C$, and $V$ denote the density, specific heat capacity, and volume of the conductor, respectively.

Formula (6) shows that if the total thermal resistance is determined, the current $I$ can be calculated from the temperature rise of the conductor.

\section{Calculation Method of Total Thermal Resistance and Current}

The total thermal resistance is composed of conduction thermal resistance a, b, c, and d inside the cavity and external convection thermal resistance $e$ and $f$. The conduction thermal resistance is determined only by the size and material of the cavity. The calculation of convection thermal resistance $\mathrm{e}$ and $\mathrm{f}$ is introduced below.

The finite element model of the measuring cavity is established. According to the numerical model of the first law of thermodynamics, the second type of boundary conditions is used for thermal analysis. The calculation grid is divided as shown in Figure 4(a), with a total of 6377 elements. The steady-state temperature field distribution of the cavity when the current is $94.9 \mathrm{~A}$ and the initial ambient temperature is $25^{\circ} \mathrm{C}$ is shown in Figure $4(\mathrm{~b})$. The heat flux of 


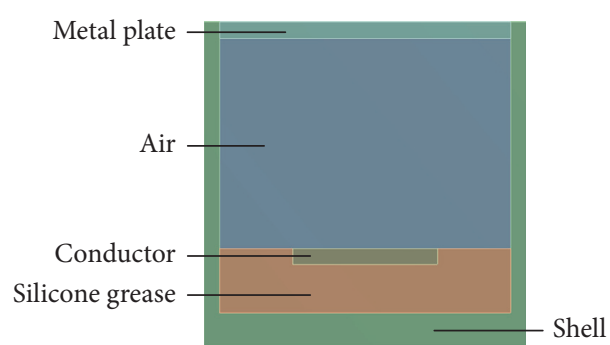

(a)

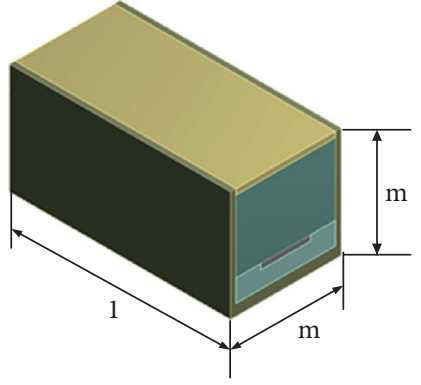

(b)

Figure 1: (a) Measuring cavity. (b) Size.

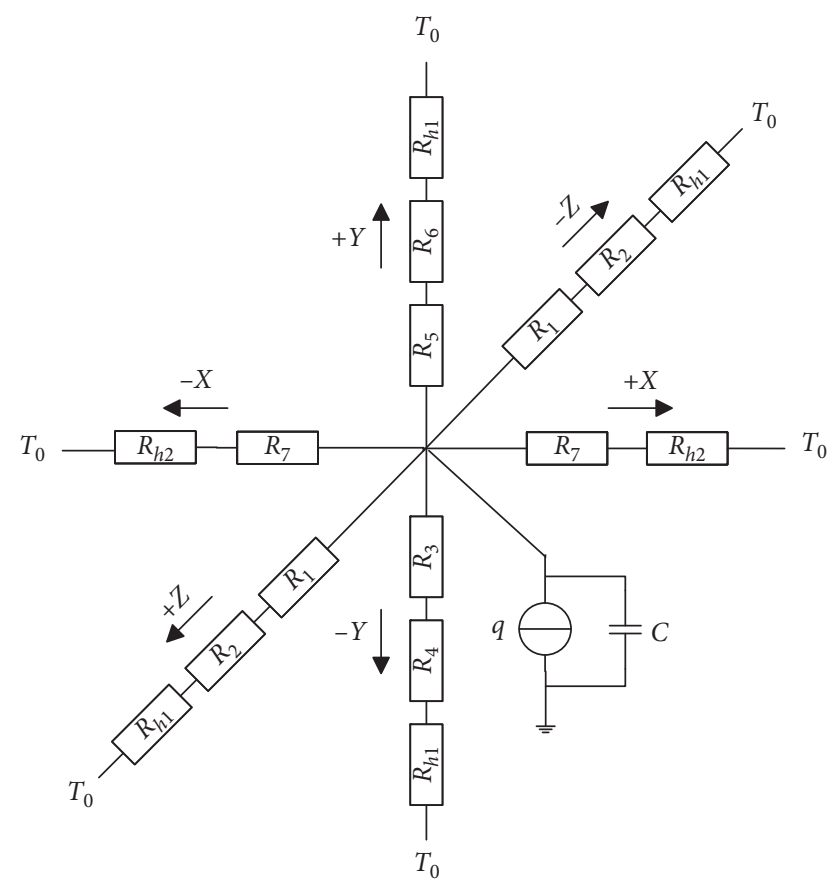

Figure 2: Thermal circuit.

TABLe 1: Symbols and meanings of thermal circuit.

\begin{tabular}{lc}
\hline Symbols & Meanings \\
\hline$R_{1}$ & Thermal resistance of silicone grease of $Z$-axis on both \\
sides of conductor
\end{tabular}

the heat exchange between the cavity and the outside is shown in Figure 5. As can be seen from Figure 4(b), the conductor has the highest temperature of $104.8^{\circ} \mathrm{C}$, while the

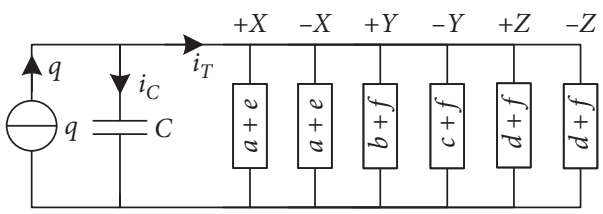

FIGURE 3: Simplified thermal circuit.

lowest temperature of $52.9^{\circ} \mathrm{C}$ appears at the upper metal plate, and the temperature distribution is symmetrical with respect to $X O Y$.

Figure 5(a) shows the heat flux of six surfaces of the cavity and Figure 5(b) shows the ratio of heat flux. The metal plate accounts for $12 \%$ of the total heat dissipation, and the lower part of the shell accounts for $22 \%$ of the total heat dissipation. Because the cavity is symmetrical with respect to the $X O Y$ and $Y O Z$ faces, the heat dissipation ratios of the outer surfaces of the $X$-axis and the $Z$-axis are the same. The heat dissipation in the two directions of the $X$-axis and the two directions of the $Z$-axis accounts for $16 \%$ and $17 \%$ of the total heat dissipation, respectively.

The convection thermal resistance can be obtained by

$$
e=\frac{1}{h \cdot A}
$$

where $h$ and $A$ denote the convection coefficient and the convection area, respectively.

The convection coefficient can be obtained by

$$
h=\frac{\Phi}{A \cdot \Delta T}
$$

where $\Phi$ and $\Delta T$ denote the heat exchange of convective heat transfer and the temperature difference of heat dissipation surface, respectively. After reaching steady state, the total heat generated by the conductor is distributed from the shell and the upper metal plate. That is, $\Phi=I^{2} R$. Thus, in the steady state,

$$
e=\frac{\Delta T}{I^{2} R} \text {. }
$$

Although all the heat generated by the conductor is distributed from the shell and the upper metal plate, the heat dissipation of the six surfaces of the cavity is not completely equal, as can be seen from Figure 5, so formula (9) can be written as follows: 


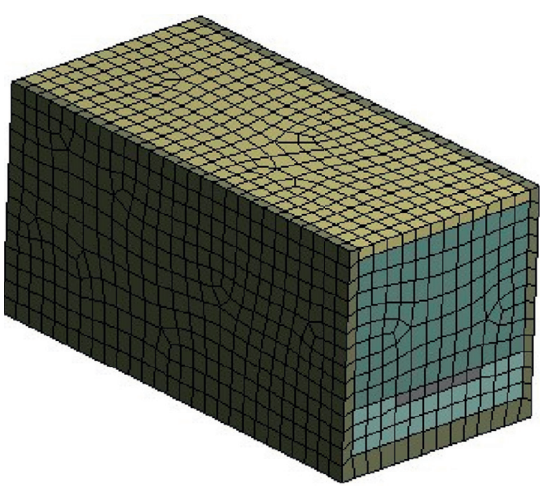

(a)

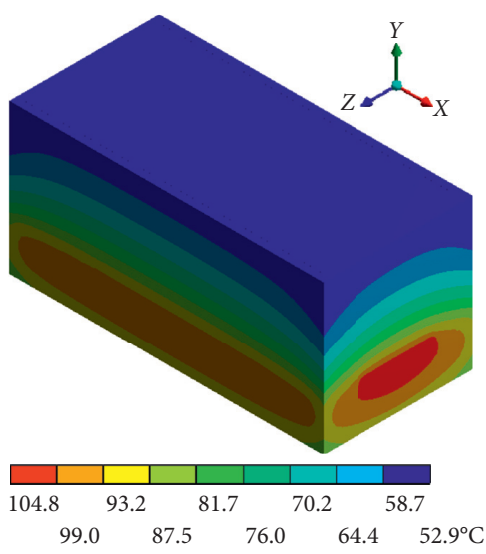

(b)

Figure 4: Finite element analysis of measuring cavity. (a) Calculation grid. (b) Temperature field of measuring cavity.

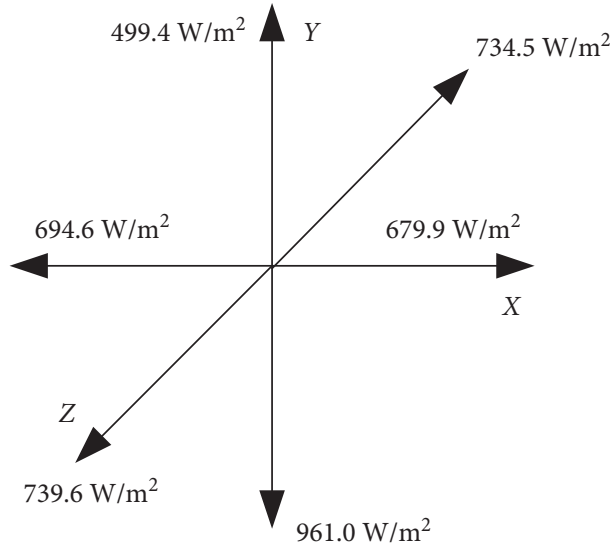

(a)

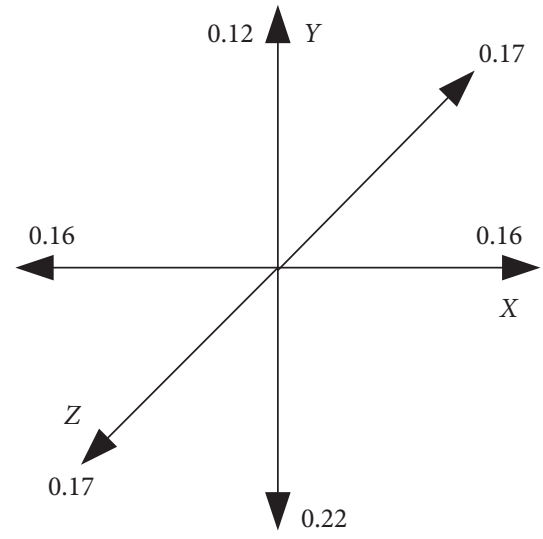

(b)

Figure 5: Heat flux of the cavity. (a) Heat flux of the shell and the metal plate. (b) Heat flux ratio.

$$
e=\frac{\Delta T}{m \cdot I^{2} R}
$$

where $m$ denotes the ratio of heat dissipation of one side to total heat $I^{2} R$. The above formula can be written as

$$
\begin{aligned}
& e_{+Y}=\frac{\Delta T_{+Y}}{m_{+Y} \cdot I^{2} R}, \\
& e_{-Y}=\frac{\Delta T_{-Y}}{m_{-Y} \cdot I^{2} R}, \\
& e_{+Z}=\frac{\Delta T_{+Z}}{m_{+Z} \cdot I^{2} R}, \\
& e_{-Z}=\frac{\Delta T_{-Z}}{m_{-Z} \cdot I^{2} R}, \\
& e_{+X}=\frac{\Delta T_{+X}}{m_{+X} \cdot I^{2} R}, \\
& e_{-X}=\frac{\Delta T_{-X}}{m_{-X} \cdot I^{2} R} .
\end{aligned}
$$

As the total heat generated by the conductor is distributed from the shell and the upper metal plate, it is obvious that $m_{+Y}+m_{-Y}+m_{+Z}+m_{-Z}+m_{+X}+m_{-X}=1$. When the structure and size of the cavity are fixed and steady state is reached, the heat dissipation ratio of the six surfaces is only related to the convection coefficient, but not to the current. In order to get the ratio, simulation is carried out on the same current and different convection coefficients. The results are shown in Table 2.

From Table 2, surfaces of $Z$-axis are always emitting $0.17 I^{2} R$, respectively. Since the cavity is symmetrical on the $X O Y$ surface, $\Delta T_{+Z}=\Delta T_{-Z}$; therefore, $m_{+Z}=m_{-Z}$. The convection thermal resistance on surfaces of $Z$-axis is

$$
e_{+Z}=e_{-Z}=\frac{\Delta T_{Z}}{0.17 \cdot I^{2} R} .
$$

Similarly, the convection thermal resistance on surfaces of $X$-axis is

$$
e_{+X}=e_{-X}=\frac{\Delta T_{X}}{0.16 \cdot I^{2} R} .
$$


TABle 2: The proportion of heat flux under different convection coefficients.

\begin{tabular}{|c|c|c|c|c|}
\hline \multirow{2}{*}{ Convection coefficient $h\left(\mathrm{~W} / \mathrm{m}^{2} \cdot{ }^{\circ} \mathrm{C}\right)$} & \multicolumn{4}{|c|}{ Heat flux density ratio } \\
\hline & $+Y$ & $-Y$ & $\pm Z$ & $\pm X$ \\
\hline 6 & 0.14 & 0.20 & 0.17 & 0.16 \\
\hline 10 & 0.13 & 0.21 & 0.17 & 0.16 \\
\hline 14 & 0.12 & 0.22 & 0.17 & 0.16 \\
\hline 18 & 0.11 & 0.23 & 0.17 & 0.16 \\
\hline 22 & 0.10 & 0.24 & 0.17 & 0.16 \\
\hline
\end{tabular}

The convective thermal resistance is only related to the convection coefficient and the heat dissipation area, and areas of the heat dissipation surfaces of $Y$ and $Z$ axes are the same, so $e_{+Y}=e_{-Y}=e_{+Z}=e_{-Z}$. And then,

$$
\frac{m_{+Y}}{m_{-Y}}=\frac{\Delta T_{+Y}}{\Delta T_{-Y}} .
$$

Formula (14) shows that the heat dissipation of the surfaces of $Y$-axis will be distributed according to the temperature rise. As can be seen from Table 2, the surfaces of $Y$-axis share a total of $0.34 I^{2} R$. So

$$
\left\{\begin{array}{l}
m_{+Y}+m_{-Y}=0.34, \\
\frac{m_{+Y}}{m_{-Y}}=\frac{\Delta T_{+Y}}{\Delta T_{-Y}} .
\end{array}\right.
$$

The convection thermal resistance of the surfaces of $Y$ axis is calculated as follows:

$e_{+Y}=\frac{\Delta T_{+Y}}{\left(0.34 \Delta T_{+Y} /\left(\Delta T_{+Y}+\Delta T_{-Y}\right)\right) I^{2} R}=\frac{\Delta T_{+Y}+\Delta T_{-Y}}{0.34 I^{2} R}=e_{-Y}$.
TABle 3: Temperature rise under different convection coefficients.

\begin{tabular}{lccccc}
\hline Convection coefficient $h$ & \multicolumn{5}{c}{ Temperature rise $\left({ }^{\circ} \mathrm{C}\right)$} \\
$\left(\mathrm{W} / \mathrm{m}^{2} \cdot{ }^{\circ} \mathrm{C}\right)$ & $\Delta T_{+Y}$ & $\Delta T_{-Y}$ & $\Delta T_{Z}$ & $\Delta T_{X}$ & $\theta$ \\
\hline 10 & 51.0 & 90.2 & 89.9 & 71.4 & 96.7 \\
11 & 45.1 & 83.1 & 82.9 & 64.9 & 89.7 \\
12 & 40.3 & 77.2 & 77.0 & 59.5 & 83.8 \\
13 & 36.2 & 72.1 & 71.9 & 54.9 & 78.8 \\
14 & 32.8 & 67.7 & 67.6 & 50.9 & 74.5 \\
15 & 29.9 & 63.8 & 63.7 & 47.5 & 70.7 \\
\hline
\end{tabular}

TABLE 4: The study results of temperature rise.

\begin{tabular}{lccc}
\hline Convection coefficient $h\left(\mathrm{~W} / \mathrm{m}^{2} \cdot{ }^{\circ} \mathrm{C}\right)$ & \multicolumn{3}{c}{ Division } \\
& $\theta / \Delta T_{+Y}$ & $\Delta T_{+Y} / \Delta T_{-Y}$ & $\theta / \Delta T_{X}$ \\
\hline 10 & 1.9 & 0.57 & 1.35 \\
11 & 2.0 & 0.54 & 1.38 \\
12 & 2.1 & 0.52 & 1.41 \\
13 & 2.2 & 0.50 & 1.44 \\
14 & 2.3 & 0.48 & 1.46 \\
15 & 2.4 & 0.47 & 1.49 \\
\hline
\end{tabular}

However,

$$
\frac{1}{R_{T}}=\frac{2}{a+e}+\frac{1}{b+f}+\frac{1}{c+f}+\frac{2}{d+f} .
$$

The expression of temperature rise-current can be obtained:

$$
\left\{\begin{array}{l}
\theta=\frac{1-e^{-(t / 1.24) \cdot I^{2} \cdot 3.31 \times 10^{-4} \cdot A}}{A}, \\
A=\frac{0.34}{\Delta T_{+Y}+\Delta T_{-Y}+0.017 I^{2}}+\frac{0.34}{\Delta T_{+Y}+\Delta T_{-Y}+0.007 I^{2}}+\frac{0.34}{\Delta T_{Z}+0.00158 I^{2}}+\frac{0.32}{\Delta T_{X}+0.00041 I^{2}} .
\end{array}\right.
$$

It is known from formula (18) that if the temperature rise of the conductor and the six surfaces is measured, the current value can be calculated. But obviously many thermistors are needed for measurement. Too many thermistors will affect the practicability of the method, so it is necessary to reduce the use of thermistors. Assuming that only $\theta$ and $\Delta T_{+Y}$ are known, the temperature rise of the conductor and the four surfaces (the $-Z$ and $-X$ temperature rise equals the $+Z$ and $+X$ temperature rise, respectively) with different convection coefficients and incoming $94.9 \mathrm{~A}$ current are simulated, and the results in Table 3 are obtained.

From Table 3 , it can be seen that $\Delta T_{-Y} \approx \Delta T_{Z}$.

In order to express $\Delta T_{-Y}$ with $\theta$ and $\Delta T_{+Y}$, the results of Table 4 are obtained.
The following can be obtained from Table 4:

$$
\begin{gathered}
\frac{\theta}{\Delta T_{X}}=0.3 \times \frac{\theta}{\Delta T_{+Y}}+0.78, \\
\frac{\theta}{\Delta T_{+Y}}=-5 \times \frac{\Delta T_{+Y}}{\Delta T_{-Y}}+4.7 .
\end{gathered}
$$

Therefore, the temperature rise of the surface of $X$ axis and the surface of $-Y$ axis can be expressed as

$$
\begin{aligned}
\Delta T_{X} & =\frac{\theta \times \Delta T_{+Y}}{0.78 \times \Delta T_{+Y}+0.3 \times \theta}, \\
\Delta T_{-Y} & =\frac{5 \times \Delta T_{+Y}}{4.7-\left(\theta / \Delta T_{+Y}\right)} .
\end{aligned}
$$


The calculation method of current $I$, which is determined only by the temperature rise of surface of $+Y$-axis $\Delta T_{+Y}$ and the temperature rise of the conductor $\theta$, is obtained

$$
\left\{\begin{array}{l}
\theta=\frac{1-e^{-(t / 1.24) \cdot I^{2} \cdot 3.31 \times 10^{-4} \cdot A}}{A} \\
A=\frac{0.34}{\Delta T_{+Y}+\left(\left(5 \times \Delta T_{+Y}\right) /\left(4.7-\left(\theta / \Delta T_{+Y}\right)\right)\right)+0.017 I^{2}}+\frac{0.34}{\Delta T_{+Y}+\left(\left(5 \times \Delta T_{+Y}\right) /\left(4.7-\left(\theta / \Delta T_{+Y}\right)\right)\right)+0.007 I^{2}} \\
+\frac{0.34}{\left(\left(5 \times \Delta T_{+Y}\right) /\left(4.7-\left(\theta / \Delta T_{+Y}\right)\right)\right)+0.00158 I^{2}}+\frac{0.32}{\left(\left(\theta \times \Delta T_{+Y}\right) /\left(0.78 \times \Delta T_{+Y}+0.3 \times \theta\right)\right)+0.00041 I^{2}}
\end{array}\right.
$$

\section{Experimental Verification}

In order to verify the correctness of the current calculation method, experimental verification was carried out. The experimental devices are shown in Figure 6.

The voltage regulator was connected to the primary side of the transformer, and the secondary side of the transformer was connected with the cavity through wires. The output current of the secondary side was measured with a clip-on multimeter, that is, the input current of the cavity. The Pt100 thermistors were fixed at the temperature measuring point (conductor and upper metal plate) by using thermal conductive double-sided adhesive. The measuring signal was sent to the computer with the thermistor temperature transmitter, and the temperature value of the measurement was read by LabVIEW.

According to the above theoretical analysis, the heat generated by the conductor in the cavity should be emitted on the shell and the upper metal plate. In order to reduce the influence of metal material on the heat dissipation of the conductor in the cavity, nylon screws and nuts were used to fix the cavity. In addition, the effect of external wires on the cavity should be reduced. It is learned that the longer the external wires are, the more the Joule heat generated by the external wires into the cavity will be which results in errors. Therefore, redundant copper sheets were inserted into the compression joint to increase the heat dissipation area of the connection and form a "heat dissipation barrier" to make the Joule heat generated by the external wires unable to transmit into the cavity to reduce error. The cavity is shown in Figure 7.

The temperature of the environment, the upper metal plate, and the conductor under different currents were measured, as shown in Table 5.

For the convenience of research, the finite element simulation model is simplified. There is no conductor outside the cavity in the finite element model. The conductor length in the experiment is $100 \mathrm{~mm}$ and which in the finite element simulation is $40 \mathrm{~mm}$. Therefore, the conductor resistance $R_{1}$ in the experiment is 2.5 times that of the finite element simulation resistance $R_{2}$. Use definition of current effective value to be equivalent:

$$
I_{1}^{2} R_{1}=I_{2}^{2} R_{2}
$$

where $I_{1}$ and $I_{2}$ denote the experimental current and the finite element simulation current, respectively. The conversion results of $I_{1}$ and $I_{2}$ are shown in Table 6 .

The simulation temperature of the environment, the upper metal plate, and the conductor under different current conditions are shown in Table 7.

The temperature data of experiment and simulation are shown in Figure 8. It can be seen that the error meets the accuracy requirement.

For the upper metal plate, when the simulation current is greater than $60 \mathrm{~A}$, the simulated temperature value will be greater than the experimental value, while for the conductor, the simulated temperature under all current is less than the experimental value. No matter the upper metal plate or the conductor, the change trend of temperature with current is the same in simulation and experiment.

Using the experimental data and MATLAB to solve the temperature rise-current equation, the calculated and the simulation current value are shown in Table 8 . The relationship between the calculation error and the simulation current is shown in Figure 9.

Table 8 and Figure 9 show that the calculation error is less than $5 \%$. The main reason for the error is that the temperature rise-current formula is deduced from the simulation data, while the calculated current value in the table is calculated from the experimental data.

The error sources of simulation data (temperature rise) and experiment data (temperature rise) are mainly as follows: the thermal conductive silicone grease in simulation is an ideal thermal conductor, but it is inevitable that there will be bubbles in the coating process of the thermal conductive silicone grease in experiment, which makes the thermal conductivity cannot be completely equivalent to the finite element simulation. In addition, the "heat dissipation barrier" cannot completely prevent the heat generated by the external wire from passing into the cavity, causing errors.

When the expanded uncertainty of $\mathrm{Pt} 100$ is $95 \%, \mathrm{U}_{95}$ is $0.026^{\circ} \mathrm{C}[14]$. The temperature measurement device based on Pt100 has a maximum temperature resistance of $300^{\circ} \mathrm{C}$, and 


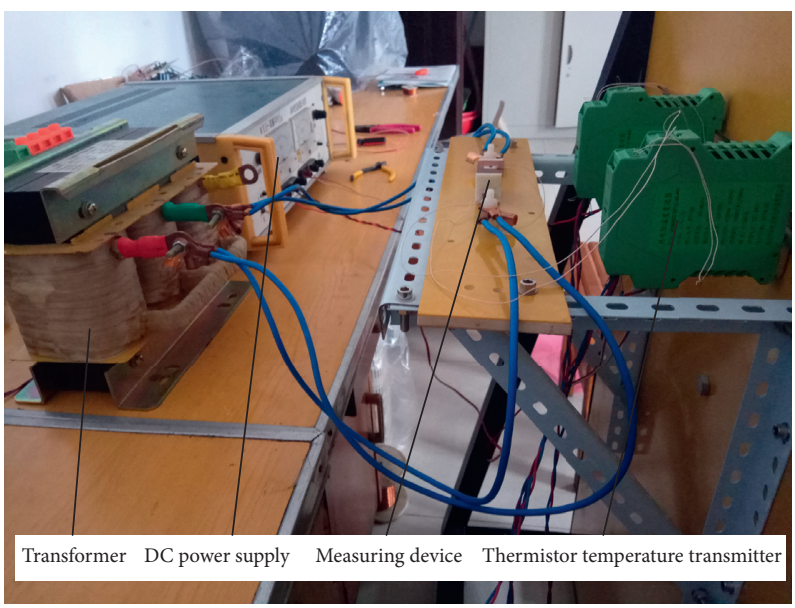

Figure 6: Experimental devices.

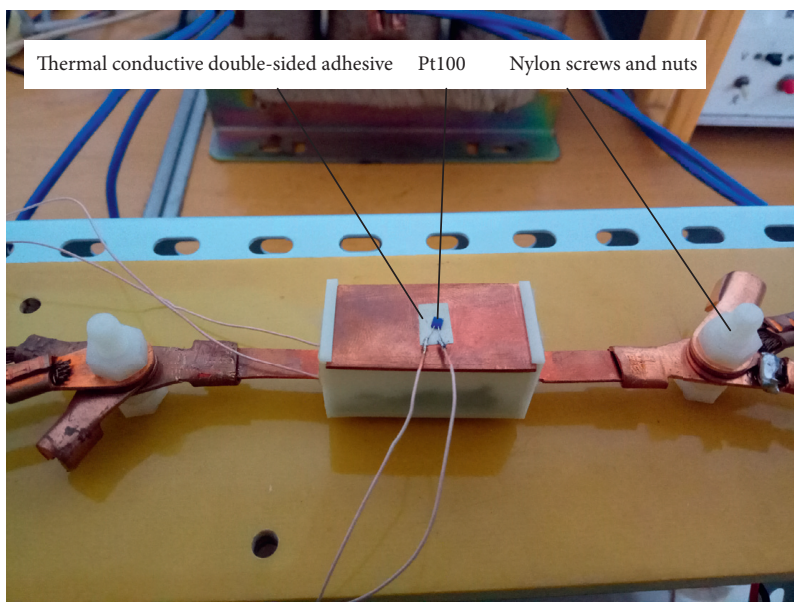

FIgURe 7: Cavity.

TABLE 5: The temperature measured by experiment.

\begin{tabular}{lccr}
\hline$I_{1}(\mathrm{~A})$ & & Temperature $\left({ }^{\circ} \mathrm{C}\right)$ & \\
Upper metal plate & Conductor \\
\hline 20 & 25.2 & 27.8 & 36.5 \\
30 & 25.9 & 31.4 & 51.5 \\
40 & 24.6 & 34.4 & 64.3 \\
50 & 24.5 & 39.2 & 84.3 \\
60 & 25.3 & 45.7 & 103.3 \\
\hline
\end{tabular}

TABle 6: Conversion of experimental current and simulation current.

\begin{tabular}{cc}
\hline$I_{1}(\mathrm{~A})$ & $I_{2}(\mathrm{~A})$ \\
\hline 20 & 31.623 \\
30 & 47.434 \\
40 & 63.246 \\
50 & 79.057 \\
60 & 94.868 \\
\hline
\end{tabular}

the conductor temperature reaches $291^{\circ} \mathrm{C}$ when the simulation current is $173.925 \mathrm{~A}$ (the experimental current is $110 \mathrm{~A})$. Therefore, the maximum current of $110 \mathrm{~A}$ can be obtained based on the size of the measuring cavity in this paper. The accuracy of the thermal resistance temperature transmitter and Pt100 used in this paper can reach $0.001^{\circ} \mathrm{C}$. When the simulation current is $0.5 \mathrm{~A}$ (the experimental current is about $0.3 \mathrm{~A}$ ), the temperature rise of the upper 
TABLE 7: Simulation results.

\begin{tabular}{ccccc}
\hline & Current $(\mathrm{A})$ & & \multicolumn{2}{c}{ Temperature $\left({ }^{\circ} \mathrm{C}\right)$} \\
Experimental & Simulation & Environment & Metal plate & Conductor \\
\hline 20 & 31.623 & 25.0 & 27.6 & 33.5 \\
30 & 47.434 & 25.0 & 35.9 & 44.1 \\
40 & 63.246 & 25.0 & 41.3 & 78.9 \\
50 & 79.057 & 25.0 & 48.5 & 101.3 \\
60 & 94.868 & 25.0 & & \\
\hline
\end{tabular}

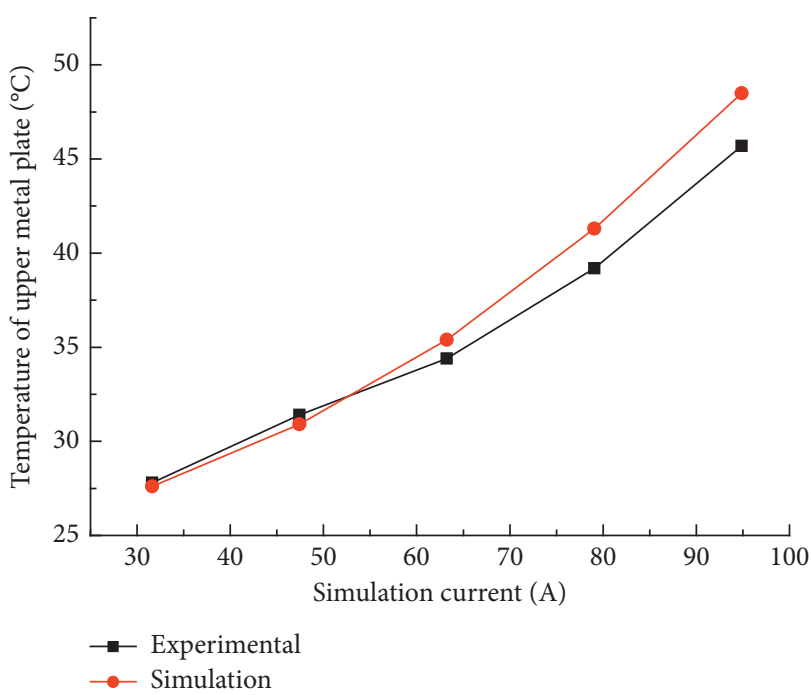

(a)

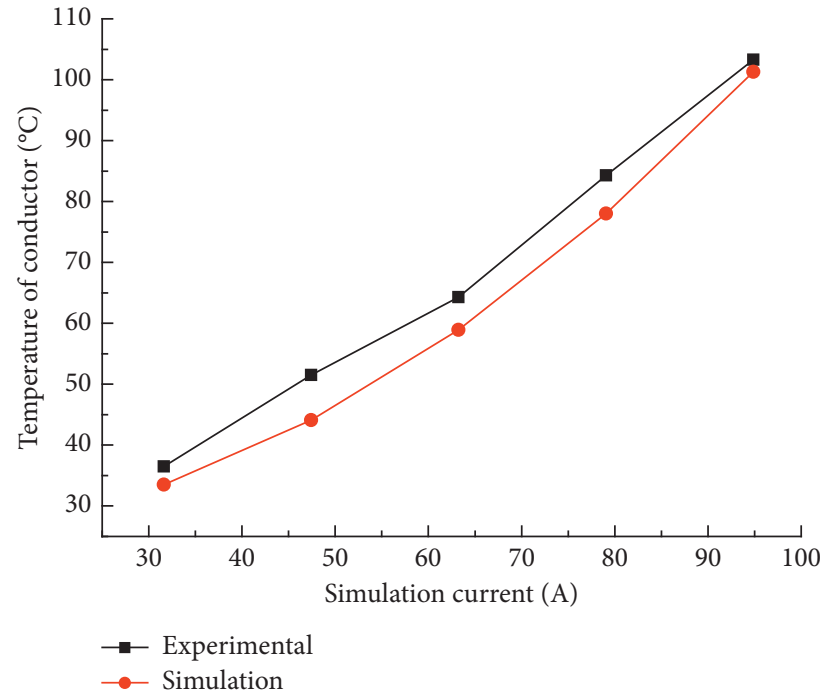

(b)

Figure 8: Contrast of data between experiment and simulation. (a) Temperature contrast of upper metal plate. (b) Temperature contrast of conductor.

TABLE 8: Calculated and simulation current values.

\begin{tabular}{lc}
\hline Calculated current $(\mathrm{A})$ & Simulation current $(\mathrm{A})$ \\
\hline 31.623 & 31.73 \\
47.434 & 47.52 \\
63.246 & 63.35 \\
79.057 & 79.19 \\
94.868 & 94.97 \\
\hline
\end{tabular}

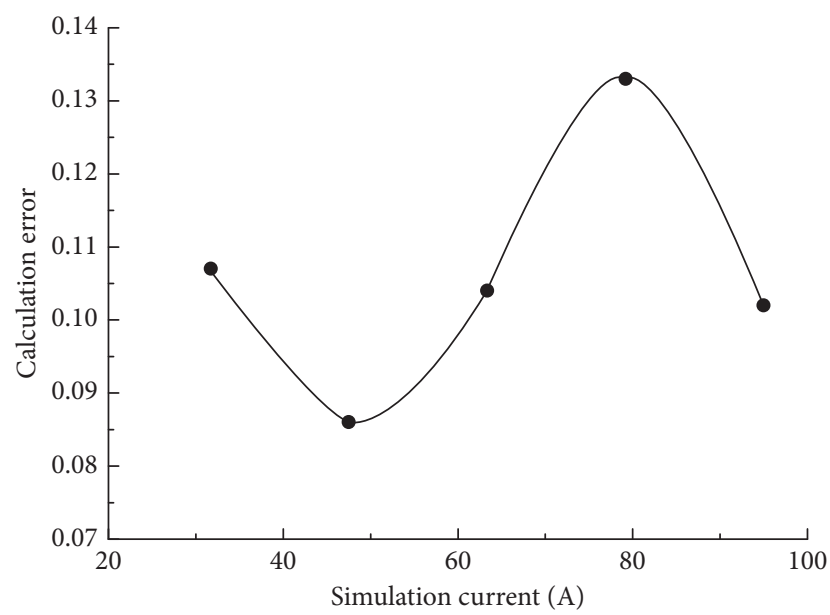

FIGURE 9: Relationship between calculation error and simulation current. metal plate is $0.001^{\circ} \mathrm{C}$. Therefore, the minimum current of $0.3 \mathrm{~A}$ can be obtained based on the size of the measuring cavity in this paper. In fact, this method will be mainly used in the future intelligent MCB overload protection, so it is more concerned about the maximum calculated current. The maximum calculated current of $110 \mathrm{~A}$ can meet the requirements of $\mathrm{MCB}$ overload protection with a rated current of $60 \mathrm{~A}$.

\section{Conclusion}

In this paper, a current calculation method based on temperature rise is introduced. Its characteristics are as follows: (1) The current is calculated based on temperature rise which can solve the core saturation problem of current transformer at high current. (2) The current calculation method does not contain the convection coefficient $h$, that is, this method is not affected by the convection coefficient. The current calculation method based on temperature rise in this paper provides a new idea for the intellectualization of MCB. The method in this paper is only applicable to the intelligent MCB with electrothermal current calculation method based on temperature rise. If the working environment changes or the size of the measuring cavity becomes larger, other current algorithms based on temperature rise should be studied. 


\section{Data Availability}

The experimental data used to support the findings of this study are included within the article.

\section{Conflicts of Interest}

The authors declare that there are no conflicts of interest regarding the publication of this paper.

\section{Acknowledgments}

This work was supported by the National Natural Science Foundation of China under the project Grant no. 51777129.

\section{References}

[1] Q. Wang, J. Xia, X. Liu, Y. Zhao, J. Li, and H. Hu, "A novel current sensor based on magnetic fluid and fiber loop cavity ring-down technology," IEEE Sensors Journal, vol. 15, no. 11, pp. 6192-6198, 2015.

[2] $\mathrm{Z}$. Wu, "A wide linearity range current sensor based on piezoelectric effect," IEEE Sensors Journal, vol. 17, no. 11, pp. 3298-3301, 2017.

[3] A. Bernieri, L. Ferrigno, M. Laracca, and A. Rasile, "An AMRbased three-phase current sensor for smart grid applications," IEEE Sensors Journal, vol. 17, no. 23, pp. 7704-7712, 2017.

[4] S. J. Nibir and B. Parkhideh, "Magnetoresistor with planar magnetic concentrator as wideband contactless current sensor for power electronics applications," IEEE Transactions on Industrial Electronics, vol. 65, no. 3, pp. 2766-2774, 2018.

[5] X. G. Yang, W. Guo, C. C. Li et al., "A fluxgate current sensor with a U-shaped magnetic gathering shell," IEEE Transactions on Magnetics, vol. 51, Article ID 4005504, 2015.

[6] W. He, J. T. Zhang, C. W. Qu, J. Wu, and J. C. Peng, "A passive electric current sensor based on ferromagnetic invariant elastic alloy, piezoelectric ceramic, and Permalloy yoke," IEEE Transactions on Magnetics, vol. 52, Article ID 4001504, 2016.

[7] P. Ripka, V. Grim, and V. Petrucha, "A busbar current sensor with frequency compensation," IEEE Transactions on Magnetics, vol. 53, Article ID 4000505, 2017.

[8] J. Han, H. Hu, H. Wang et al., "Temperature-compensated magnetostrictive current sensor based on the configuration of dual fiber Bragg gratings," Journal of Lightwave Technology, vol. 35, no. 22, pp. 4910-4915, 2017.

[9] S. Y. Xu, F. F. Xing, W. Li et al., "A stray current sensor based on an all-side cylindrical spiral fiber," IEEE Photonics Journal, vol. 9, Article ID 6800814, 2017.

[10] H. Zhang, "High temperature and vibration robustness allfiber current sensor with a fiber-loop architecture and reflection scheme," IEEE Photonics Journal, vol. 9, Article ID 7103808, 2017.

[11] Y. P. Liu, L. Ma, and Z. Y. He, "Birefringence variation independent fiber-optic current sensor using real-time SOP measurement," IEEE Photonics Journal, vol. 9, Article ID $7105409,2017$.

[12] P. Wei, C. Cheng, and T. Liu, "A photonic transducer-based optical current sensor using back-propagation neural network," IEEE Photonics Technology Letters, vol. 28, no. 14, pp. 1513-1516, 2016.

[13] J. Wu, Z. Chen, C. Wang, and L. Hao, "A novel low-cost multicoil-based smart current sensor for three-phase currents sensing of overhead conductors," IEEE Transactions on Power Delivery, vol. 31, no. 6, pp. 2443-2452, 2016.
[14] Z. Sun, H. Z. Wang, Y. N. Liu, and H. Li, "Uncertainty evaluation of verification results of platinum resistance temperature sensor," Technology Innovation and Application, vol. 25, pp. 16-18, 2017. 